\title{
Effect of Berberine on Hyperuricemia and Kidney Injury: A Network Pharmacology Analysis and Experimental Validation in a Mouse Model
}

\author{
Qiaoping $\mathrm{Li}^{1, *}$ \\ Ziwei Huang ${ }^{2, *}$ \\ Defu Liu ${ }^{3}$ \\ Jingna Zheng' \\ Jianhui $\mathrm{Xie}^{4-6}$ \\ Jiannan Chen' \\ Huifang Zeng $\mathbb{D}^{2}$ \\ Ziren Su' \\ Yucui $\mathrm{Li}^{\mathrm{I}}$

\begin{abstract}
'School of Pharmaceutical Sciences, Guangzhou University of Chinese Medicine, Guangzhou, 510006, People's Republic of

China; ${ }^{2}$ The First Affiliated Hospital of Chinese Medicine, Guangzhou University of Chinese Medicine, Guangzhou, 510405 , People's Republic of China; ${ }^{3}$ School of Basic Medical Sciences, Guangzhou University of Chinese Medicine, Guangzhou, 510006 , People's Republic of China; ${ }^{4}$ The Second Affiliated Hospital of Guangzhou University of Chinese Medicine, Guangzhou, 510120 , People's Republic of China; ${ }^{5}$ State Key Laboratory of Dampness Syndrome of Chinese Medicine, The Second Affiliated Hospital of Guangzhou University of Chinese Medicine, Guangzhou, 510120 , People's Republic of China; ${ }^{6}$ Guangdong Provincial Key Laboratory of Clinical Research on Traditional Chinese Medicine Syndrome, Guangzhou, 5I0I20, People's Republic of China
\end{abstract}

*These authors contributed equally to this work

Correspondence: Yucui Li; Ziren Su Guangzhou University of Chinese Medicine, Guangzhou, 510006, People's Republic of China

Tel +862039358517

Fax +862039358390

Email liyucui@gzucm.edu.cn;

suziren@gzucm.edu.cn
Purpose: Berberine (BBR) is an active component of Phellodendri Cortex (PC), which is a traditional Chinese medicine that has been prescribed clinically for hyperuricemia (HUA) for hundreds of years. Many studies reported the anti-inflammatory and nephroprotective properties of BBR and PC; however, the therapeutic effects of BBR on HUA have not been explored. This study aims to investigate the efficacy and mechanism of BBR for treating HUA.

Methods: The mechanism of BBR in the treatment of HUA were predicted by network pharmacology. A mouse model of HUA established by potassium oxonate and hypoxanthine was used to verify the prediction. The levels of serum uric acid (UA), urea nitrogen (BUN) and creatinine (CRE) were determined by biochemical test kits. Hematoxylin and eosin staining of kidney tissues was used to observe the kidney damage. ELISA kits were applied to detect the levels of interleukin (IL)-1 $\beta$ and IL-18 in serum and kidney tissues. Quantitative real-time PCR and Western blotting were adopted to analyze the expression of NLRP3, ASC, Caspase1, IL-1 $\beta$ and URAT1. The expressions of URAT1 in the kidney tubules were visualized by immunohistochemical staining. Molecular docking was used to assess the interaction between URAT1 and BBR.

Results: The network pharmacology screened out 82 genes and several inflammation-related signaling pathways related to the anti-hyperuricemia effect of BBR. In the in vivo experiment, BBR substantially decreased the level of UA, BUN and CRE, and alleviated the kidney damage in mice with HUA. BBR reduced IL-1 $\beta$ and IL-18, and downregulated expressions of NLRP3, ASC, Caspase1 and IL-1 $\beta$. BBR also inhibited expression of URAT1 and exhibited strong affinity with this target in silico docking.

Conclusion: BBR exerts anti-HUA and nephroprotective effects via inhibiting activation of NLRP3 inflammasome and correcting the aberrant expression of URAT1 in kidney. BBR might be a novel therapeutic agent for treating HUA.

Keywords: berberine, hyperuricemia, URAT1, NLRP3 signaling pathway

\section{Introduction}

Hyperuricemia (HUA) is a potentially harmful condition involving high uric acid (UA) serum levels $(>7.0 \mathrm{mg} / \mathrm{dL})^{1}$ induced by purine metabolism disorders. The prevalence of HUA is increasing, and it is therefore becoming an important problem. $^{2}$ It is likely to become even more prevalent in the future due to unhealthy modern lifestyles. HUA is frequently asymptomatic, but it contributes to the development of gout and kidney disease, ${ }^{3,4}$ and is closely associated with cardiovascular diseases ${ }^{5}$ and various metabolic diseases. ${ }^{6}$ Notably, increased UA and the 
formation of monosodium UA crystals can both cause severe inflammatory responses, leading to kidney injury without spontaneous remission. ${ }^{7,8}$

For HUA treatment, it is also important to reduce the UA production and promote excretion of UA in patients. However, current anti-HUA reagents often also cause adverse effects. Xanthine oxidase (XOD) is known as a pivotal enzyme which plays an important part in UA formation. ${ }^{9}$ Allopurinol, an XOD inhibitor, is a major therapeutic option but causes various side effects, such as severe cutaneous adverse reactions and Stevens-Johnson syndrome. ${ }^{10}$ Benzbromarone (BZB) targets renal organic anion transporters that regulate UA excretion to exert an anti-HUA effect. However, the clinical application of BZB is hampered by its kidney toxicity. ${ }^{11}$ Febuxostat is a novel and more efficacious XOD inhibitor; however there is evidence demonstrating its relationship with cardiovascular diseases. ${ }^{12}$ Although a recent clinical trial did not support this finding, ${ }^{13}$ its cardiovascular safety is still controversial. $^{14}$ Therefore, it is very urgent to find a more effective and safer candidate for treating HUA.

Phellodendri Cortex (PC), known as Huangbai in traditional Chinese medicine, has been applied in classical formulas, such as Simiao powder, Jinhuang cream and Tongfeng decoction, to treat gout for hundreds of years. ${ }^{15}$ Research has demonstrated that PC and these formulas exert anti-gout effects by relieving inflammation and reducing UA. ${ }^{16}$ The pharmacological mechanism may be closely related to certain chemical ingredients derived from them. Berberine (BBR, Figure 1A), a major alkaloid in $\mathrm{PC}$, has been considered as a promising compound for ameliorating HUA. ${ }^{17}$

BBR exhibits various pharmacological activities and low toxicity. ${ }^{18}$ Several studies have indicated that BBR has many positive effects on HUA. A clinical trial showed that BBR could reduce UA in patients with difference complications. ${ }^{19}$ BBR combined with benzbromarone, ${ }^{20}$ amlodipine $^{21,22}$ or silymarin ${ }^{23}$ did not only lower UA level but also regulate metabolism disorders and reduce blood pressure. The hypouricemic effect of BBR was further demonstrated on a rat model, ${ }^{24}$ and might be associated with urate transporter 1 (URAT1) which is a key factor regulating the UA level in kidney. ${ }^{25}$

Hyperuricemia has been recognized as a potential risk factor for kidney injury. ${ }^{3,4}$ Emerging evidence from animal and clinical studies validates that BBR has an outstanding nephroprotective effect. Previous researches have shown that BBR suppresses NLRP3 inflammasome activation and therefore alleviates inflammation induced by UA crystal in rat and cell models. ${ }^{26,27}$ The pronounced antiinflammatory effects of BBR might contribute to its beneficial effects against acute and chronic kidney injury. ${ }^{28-30}$

In summary, BBR has anti-hyperuricemia and nephroprotective potential; however, its therapeutic effects on HUA and underlying mechanisms are still incomplete and confused. To fill this gap in the literature, we predicted the anti-HUA efficacy and mechanisms of BBR via network pharmacology and validated our hypothesis in mice with potassium oxonate (PO)- and hypoxanthine (HX)-induced HUA. The results show that high-dose BBR lowers the serum uric acid (UA), blood urea nitrogen (BUN), creatinine (CRE), and proinflammatory cytokine (interleukin [IL]-1 $\beta$ and IL-18) levels in vivo. This study further reveals the mechanisms underlying the anti-HUA and nephroprotective effects of BBR, which involve suppressing NLRP3 inflammasome activation and inhibiting the expression of URAT1. These findings provide evidence that BBR might be an effective UA-lowering and nephroprotective agent for treating HUA.

\section{Materials and Methods Chemical Reagents}

Potassium oxonate (PO, high-performance liquid chromatography [HPLC] purity $\geq 98 \%$ ), hypoxanthine (HX, HPLC purity $\geq 98 \%$ ), and benzbromarone (BZB, HPLC purity $\geq 98 \%$ ) were supplied by Yuan-Ye Biotechnology Co., Ltd. (Shanghai, China). Berberine (BBR, HPLC purity $\geq 98 \%$ ) was obtained from Meilun Biotechnology Co., Ltd. (Dalian, China). Pentobarbital sodium was provided by Shanghai Harvest Pharmaceutical Co., Ltd. (Shanghai, China).

The assay kits for UA, BUN, and CRE were obtained from Jiancheng Biotechnology Co., Ltd. (Nanjing, China). Enzyme-linked immunosorbent assay (ELISA) kits for IL18 and IL-1 $\beta$ ) were bought from Mlbio Co. Ltd. (Shanghai, China). Mouse gene primers were obtained from Sangon Biological Engineering Co., Ltd. (Shanghai, China). TRIzol ${ }^{\circledR}$ reagent was obtained from Thermo Fisher (Waltham, MA, USA). The HiScript II Q RT SuperMix kit and the ChamQ ${ }^{\mathrm{TM}} \mathrm{SYBR}^{\circledR}$ qPCR Master Mix kit were provided by Vazyme Biotech Co., Ltd. (Nanjing, China). Primary antibodies against NLRP3, IL-1 $\beta$ and $\beta$-actin were obtained from Abcam (Cambridge, UK), primary antibodies against ASC and caspase1 were obtained from Biosciences (Cincinnati, OH, USA), and primary antibodies against URAT1 was obtained from Proteintech (Wuhan, China). A secondary antibody for Western 
A<smiles>COc1ccc2cc3[n+](cc2c1OC)CCc1cc2c(cc1-3)OCO2</smiles>

B
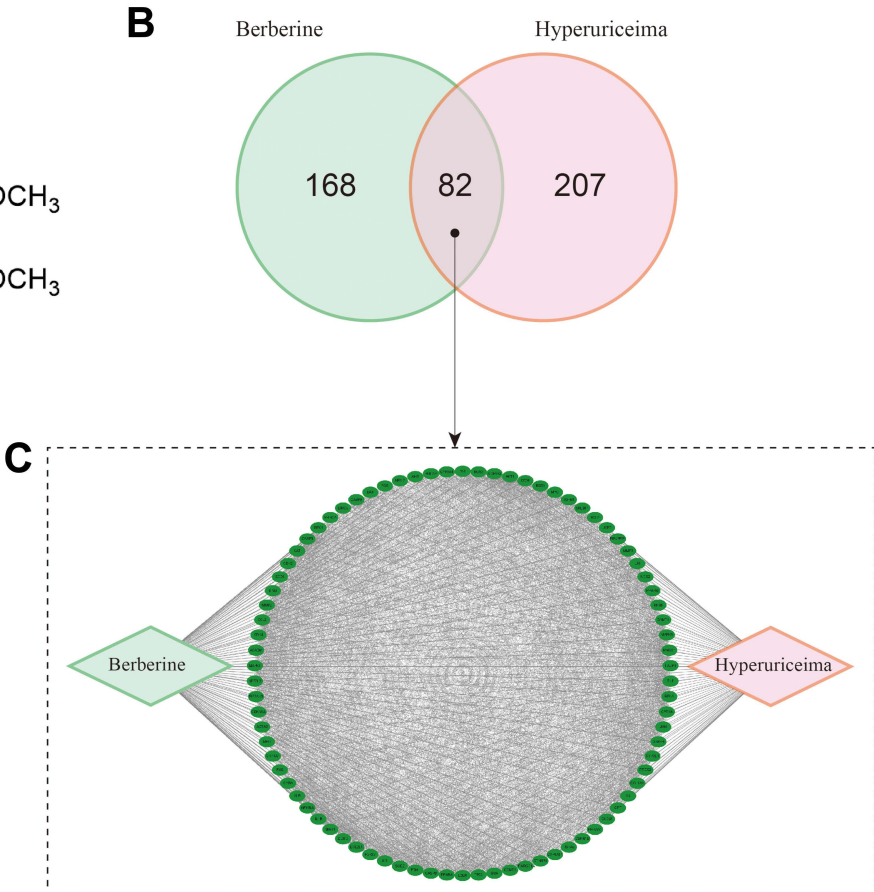

Figure I The network pharmacology of BBR in the treatment of HUA. (A) The chemical structure of BBR. (B) The Venn diagram of BBR-HUA. (C) The BBR-target-HUA network.

blotting was supplied by Affinity Biosciences (Cincinnati, $\mathrm{OH}, \mathrm{USA}$ ). Horseradish peroxidase (HRP)-conjugated secondary antibody for immunohistochemical staining and HRP-conjugated streptavidin were purchased from Thermo Fisher. Other reagents used in the study were of analytical or HPLC grade.

\section{Network Pharmacology}

The 250 BBR-related targets were retrieved from CTD database (http://ctdbase.org/search/) (Table S1), and 289 HUArelated disease genes were obtained from CTD (Inference Score $\geq 30$ ) and DiseGeNET databases (PLI $\geq 0.05$, https:// www.disgenet.org/) (Table S2). These two gene collections were analyzed by R programming language (version 4.0.3) to identify BBR-HUA common targets.

The protein-protein interaction (PPI) network was constructed by the STRING database (https://string-db.org/) with a high confidence level (interaction score $>0.70$ ). The BBR-target-HUA network was visualized by Cytoscape software (version 3.8.0). The core genes were screened two times by CytoNCA (a program in Cytoscape software) according to Betweenness Centrality (BC).

Functional and pathway enrichment analyses of 50 core targets, the result of the first screen (Table S4), was performed using a tool provided by OmicShare (https:// www.omicshare.com/tools/Home/Soft/getsoft) based on
GO (http://geneontology.org/) and KEGG databases (https://www.kegg.jp/kegg/pathway.html), and 20-top ranked results were displayed in a bubble chart.

\section{Animals and Treatments}

Male Institute of Cancer Research (ICR) mice (20 $\pm 2 \mathrm{~g}$, 4 weeks) were obtained from Guangdong Medical Laboratory Animal Center (Foshan, China). All ICR mice were specific-pathogen-free. The mice were fed in standard conditions at least 7 days before the experiment (constant temperature of $24 \pm 1{ }^{\circ} \mathrm{C}$ and a $12 / 12 \mathrm{~h}$ light-dark cycle). All animal experiments were approved by the Animal Experimental Ethics Committee of Guangzhou University of Chinese Medicine (no. 20200510001) and conducted in accordance with the Declaration of Helsinki (as revised in 2013).

The mice were randomly divided into five groups ( $n=10$ per group): normal negative control (Con group), HUA (model group), benzbromarone (5 mg/kg; BZB group; positive control), $50 \mathrm{mg} / \mathrm{kg}$ berberine (low-dose BBR group), and $100 \mathrm{mg} / \mathrm{kg}$ berberine (high-dose BBR group). In all groups except for the Con group, HUA was induced by $\mathrm{PO}$ and $\mathrm{HX}$, as previously reported. ${ }^{31}$ Briefly, all mice were administered intraperitoneally with PO (300 mg/ $\mathrm{kg})$ and orally with $\mathrm{HX}(300 \mathrm{mg} / \mathrm{kg})$ except those in Con group (received $0.9 \%$ physiological saline at the same volume). At $1 \mathrm{~h}$ after completing the model treatment, the 
mice were treated with the relevant drugs (BZB or BBR) or saline. The mice were administered as described above for 10 consecutive days. The dosages of BZB and BBR were based on our pilot experiment.

\section{Sample Collection and Relative Kidney Weight}

After fasted overnight, mice were anesthetized by intraperitoneal injection with $2 \%$ pentobarbital sodium $(50 \mathrm{mg} / \mathrm{kg})$ according to the guideline. ${ }^{32}$ The blood samples were taken from the abdominal aorta and then were centrifuged at $3000 \mathrm{rpm}$ at $4^{\circ} \mathrm{C}$ for $10 \mathrm{~min}$ to obtain serum. Kidneys were harvested, weighed, and cut into small pieces for the following analyses. The relative kidney weight was calculated by dividing the weight of the kidney by the body weight. All samples were preserved at $-80{ }^{\circ} \mathrm{C}$.

\section{Biochemical and Cytokine Level Analysis}

The levels of UA, BUN, and CRE in serum were measured by commercial kits. ELISA kits were used to detect the levels of IL-1 $\beta$ and IL-18 in the serum and kidney tissues. The test was performed according to the specification form manufacturer of kits.

\section{Histopathological Examination}

The kidneys were fixed in $4 \%$ paraformaldehyde and the embedded in paraffin and sliced into sections $(4 \mu \mathrm{m})$ after $24 \mathrm{~h}$. Hematoxylin and eosin (H\&E) were used to stain the kidney sections. Micropathological observation of the cortex and medulla in the kidney tissue was conducted under a light microscope (OLYMPUS, Tokyo, Japan) at 200× and $400 \times$. The kidney histopathological lesions were scored by a blinded assessor according to the criteria in Table $1 .^{33}$

\section{Quantitative Real-Time PCR}

The experiment was carried out according to the published protocols and guidelines. ${ }^{34,35}$ TRIzol $^{\circledR}$ reagent was used to obtain total RNA from the kidney samples. The RNA was reverse transcribed to cDNA by using the HiScript II Q RT SuperMix kit. The cDNA was amplified using primers as follows: URAT1 forward primer: $5^{\prime}$ - TGTCAAC AGGAGGAAGGCAGAGG-3'; URAT1 reverse primer: 5'CACAGCATGGAGATGATGGTTCGG-3'; NLRP3 forward primer: 5'-GCTGCGATCAACAGGCGAGAC-3'; NLRP3 reverse primer: 5'-AAGGCTGTCCTCCTGGCATACC-3'; ASC forward primer: 5'-GATGCCATCCTGGACGC TCTTG-3'; ASC reverse primer: 5'-GCCCATAGCCTT CTCGCAGTTG-3'; Caspase1 forward primer: 5'AGGACTGACTGGGACCCTCAAG-3'; Caspase1 reverse primer: 5'-GGGCAAGACGTGTACGAGTGG-3'; IL-1 $\beta$ forward primer: 5'-TCGCAGCAGCACATCAACAAGAG-3'; IL-1 $\beta$ reverse primer: 5'- TGCTCATGTCCTCATC CTGGAAGG-3'; $\quad \beta$-acin forward primer: $5^{\prime}$ GGTCATCACTATTGGCAACG-3'; $\beta$-acin reverse primer: 5'-ACGGATGTCAACGTCACACT-3'

The reaction conditions of cycle procedures were set at $95{ }^{\circ} \mathrm{C}$ for $10 \mathrm{~min}$, and 40 cycles at $95{ }^{\circ} \mathrm{C}$ for $15 \mathrm{~s}$ and 60 ${ }^{\circ} \mathrm{C}$ for $60 \mathrm{~s}$. $\beta$-actin was used to housekeep the expression levels of gene and the relative expression levels of target genes were calculated using the $2^{-\Delta \Delta C t}$ method.

Table I Criteria and Scores for H\&E-Stained Histopathological Lesions

\begin{tabular}{|l|r|c|}
\hline \multicolumn{2}{|l|}{ Criteria } & Score: Percent (\%) of the Visual Field for the Respective Criteria \\
\hline \multirow{2}{*}{ Glomeruli } & Damaged glomeruli & 0: No change \\
\hline \multirow{3}{*}{ Renal tubular } & Irregular contour and dilatation & I: $<15 \%$ change \\
\cline { 2 - 3 } & Brush border loss of proximal tubular cells & $2: 16-30 \%$ change \\
& & $3: 31-50 \%$ change \\
& & $4:>50 \%$ change \\
\hline \multirow{2}{*}{ Other } & Necrosis & The total amount \\
\cline { 2 - 3 } & Inflammatory cell infiltration & \\
\cline { 2 - 3 } & Crystalline-type deposit & \\
\hline
\end{tabular}

Abbreviations: BBR, Berberine; BC, Betweenness centrality; BZB, Benzbromarone; BUN, Blood urea nitrogen; CRE, Creatinine; ELISA, Enzyme-linked immunosorbent assay; HUA, Hyperuricemia; HX, Hypoxanthine; H\&E, Hematoxylin and eosin; HPLC, High-performance liquid chromatography; IL, Interleukin; NLRP3, Nucleotide binding oligomerization domain-like receptor protein 3; PMSF, phenylmethylsulfonyl fluoride; PO, Potassium oxonate; PPI, Protein-protein interaction; PVDF, polyvinylidene fluoride; RIPA, Radioimmunoprecipitation assay; TBST, Tris-buffered saline-Tween 20; URATI, Urate transporter I; UA, Uric acid; XOD, Xanthine oxidase. 


\section{Western Blotting}

The procedure of Western blotting was referred to the published protocol. ${ }^{36,37}$ Radioimmunoprecipitation assay (RIPA) lysis buffer (contained $1 \mathrm{mM}$ of the protease inhibitor phenylmethylsulfonyl fluoride [PMSF]) was used to extract kidney proteins and then loading buffer was used to denature kidney proteins. Samples of proteins were separated by $10 \%$ polyacrylamide gel and transferred to membranes of polyvinylidene fluoride (PVDF). 5\% skim milk was prepared by Tris-buffered saline-Tween 20 (TBST) and applied to block the PVDF membranes for $1 \mathrm{~h}$. Primary antibody against NLRP3, ASC, caspase1, IL-1 $\beta$, URAT1or $\beta$-actin (1:1000) was used to incubate the membranes overnight at $4^{\circ} \mathrm{C}$ after washing three times with TBST and HRPconjugated secondary antibody (1:3000) was used to incubate for $1 \mathrm{~h}$ at room temperature. An enhanced chemiluminescence kit was applicable to visualize the blots. All the results were analyzed by using ImageJ software (National Institutes of Health, MA, USA). All blots were quantified and normalized against $\beta$-actin.

\section{Immunohistochemical Staining}

The Immunohistochemical staining was conducted as described previously. ${ }^{38,39}$ The paraffin-embedded kidney sections were deparaffinized using xylene and dehydrated using graded concentrations of alcohol. The kidney samples of section were autoclaved with citrate buffer $(\mathrm{pH}$ 6.0) at $120^{\circ} \mathrm{C}$. After $15 \mathrm{~min}, 3 \%$ hydrogen peroxide was used to inactivate the endogenous peroxidase. $10 \%$ horse serum was used to block the sections and then incubated with primary antibodies against URAT1 $(1: 200)$ at $4^{\circ} \mathrm{C}$ overnight. The sections were then incubated at $37^{\circ} \mathrm{C}$ for 30 min with an HRPconjugated secondary antibody and subsequently incubated with HRP-conjugated streptavidin (Bioss, Beijing, China). A microscope was used to observe the sections and the expression of URAT1 was analyzed using ImageJ software.

\section{Molecular Docking}

The docking analysis was performed using AutoDock 4.2.6 (Scripps Research Institute, La Jolla, CA, USA). The structure of the URAT1 protein was modeled using Modeller 9.11 (https://salilab.org/modeller/) and EasyModeller 4.0 (http://modellergui.blogspot.com/) according to a previous report. ${ }^{40}$ Crystal structures of BBR were obtained from the PubChem database (https:// pubchem.ncbi.nlm.nih.gov/, CID: 2353) and used as the ligands for docking. The parameters for the docking calculation were set as previously described. ${ }^{40}$ Briefly, the protein receptor was placed in a grid box of $30 \times 30 \times 30 \AA$ with $0.375 \AA$ spacing. The binding free energy $(\mathrm{kcal} / \mathrm{mol})$ was predicted using the Lamarckian genetic algorithm to evaluate the affinity. The specific interaction between URAT1 and BBR was visualized using PyMOL (Schrödinger LLC, NY, USA).

\section{Statistical Analysis}

Statistical analysis complied with the recommendations on experimental design and analysis in pharmacology ${ }^{41}$ and undertaken only for at least five biologically independent replicates. Any data with small group sizes $(\mathrm{n}<5)$ were not subjected to statistical analysis.

Q-Q plot was used to assess data distribution (all Q-Q plots were shown in Figure S8). For data following normal distribution the ANOVA test with Fisher's least significant difference (LSD) test was used to compare different groups. $\mathrm{P}$ values $<0.05$ were considered significant. All results were analyzed by SPSS 25.0 (IBM Corp., Armonk, NY, USA) and showed as mean \pm SEM.

\section{Results}

\section{Potential Anti-HUA Effect and Its Mechanisms of BBR}

82 BBR-HUA common targets were identified (Figure 1B and $\mathrm{C}$ and Table $\mathrm{S} 3$ ). To narrow the key targets of BBR treating HUA, the PPI network was constructed and analyzed by STRING database and Cytoscape (Figure 2A). Ranked by the BC score, 15 core genes were obtained: AKT1, IL6, VEGFA, STAT3, TP53, INS, MAPK1, MAPK3, IGF1, TNF, JUN, MAPK8, MYC, IL1B, and ESR1. (Figure 2B) (Table S5).

The GO and KEGG enrichment analyses were performed to further predict the underlying mechanism of BBR in HUA. Several biological processes, such as cellular response to oxygen-containing compound and endogenous stimulus might be involved the treatment of HUA (Figure 3A). The cellular membrane components might play an important role on the mechanism of BBR on HUA (Figure 3B). The antiHUA effect of BBR was possibly related to signaling receptor binding, identical protein binding, enzyme binding, etc. (Figure $3 \mathrm{C}$ ), and its underlying signaling might be AGERAGE, TNF- $\alpha$ and IL-17 pathway (Figure 3D). Based on these results and literature review, the NLRP3 inflammation was selected as the cellular signaling we were interested and validated in HUA mice as described below (Figure 3E). 


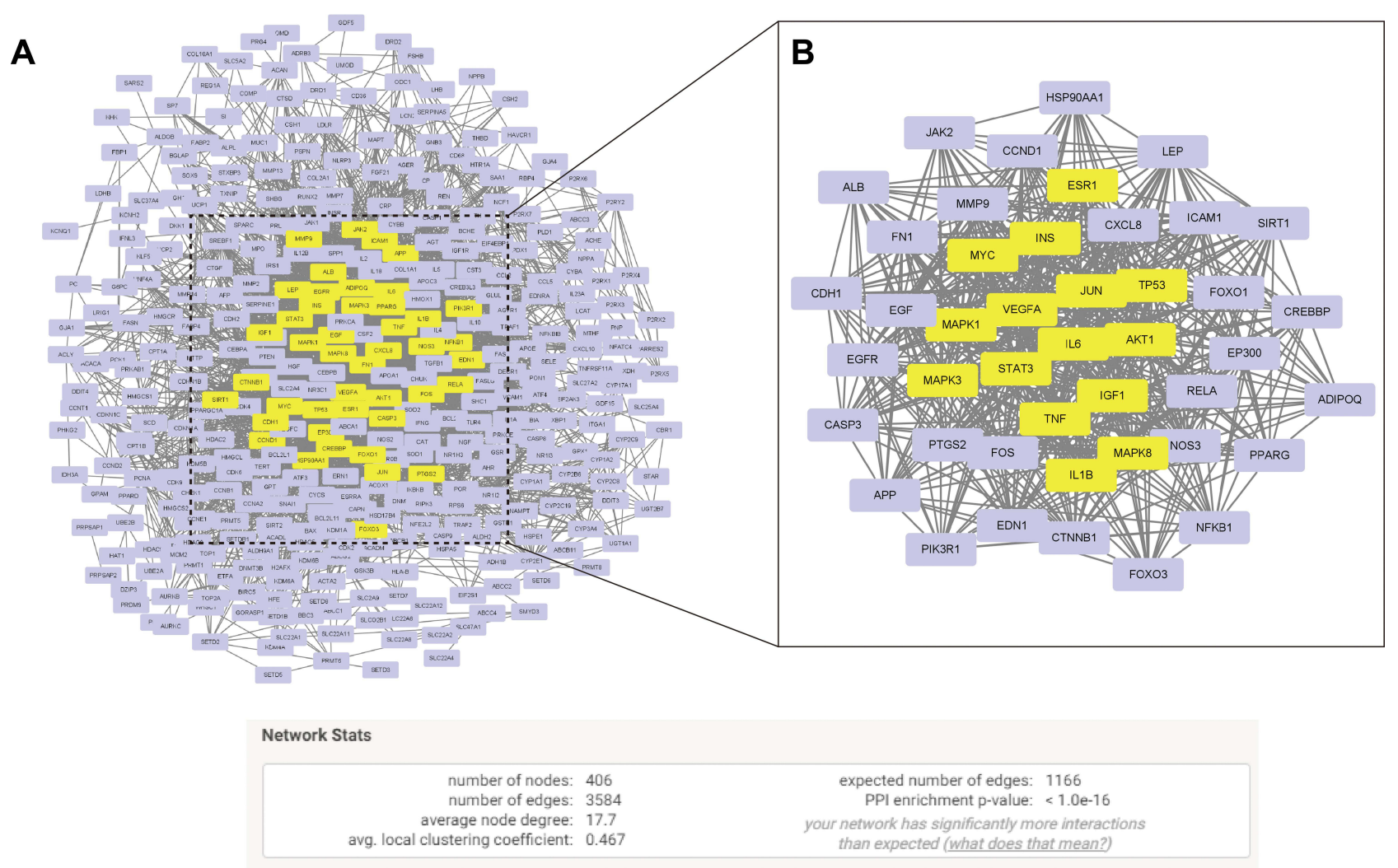

Figure 2 The potential targets though which BBR regulate HUA. The protein-protein interaction (PPI) network of 82 BBR-HUA common genes after (A) the first screen or (B) the second screen (Yellow node: core targets).

\section{BBR Decreased the Levels of UA, CRE, and BUN in Serum}

The serum level of UA was increased by PO and HX treatment compared to the level in the Con group $(\mathrm{P}<0.01)$ as shown in Figure 4, indicating the mouse model of HUA was established successfully. In the model group, body weight growth was inhibited and the kidneys were impaired, as reflected by the significantly increased serum levels of CRE and BUN compared to the levels in the Con group $(\mathrm{P}<0.01)$. BZB and different dose BBR significantly decreased the UA level in serum compared to the level in the model group, and the body weight of the mice increased stably over time in these groups $(\mathrm{P}<0.01)$. BZB and high-dose BBR also significantly obviously decreased the serum level of CRE in comparison with the level in the model group $(\mathrm{P}<0.01)$. BZB and BBR also reduced the level of BUN, but only highdose $\mathrm{BBR}$ significantly decreased it $(\mathrm{P}<0.01)$.

\section{BBR Ameliorated Kidney Histopathological Changes}

Kidney changes were observed in the model mice (Figure 5A and B). While the kidney tissues in the Con group had a normal surface and cell structure, the kidneys of the model mice were characterized by unclear boundaries, atrophied glomeruli, proximal tubules without brush borders, and occasional crystal stones in the dilated tubules. Compared to the model group, BZB and BBR alleviated these pathological lesions to various degrees. The low-dose BBR group exhibited some improvement in the proximal convoluted tubules but still exhibited severe glomerular capsule disruption and tubule dilation. However, high-dose BBR and BZB remarkably prevented the damage to the glomeruli and tubules, with intact glomeruli, regular tubules, and clear brush borders being observed. The relative kidney weight (Figure 5C) and histopathological scores (Figure 5D) were also significantly lowered by high-dose BBR and BZB in comparison with the model group $(\mathrm{P}<0.01)$.

\section{BBR Suppress NLRP3 Inflammasome Activation to Inhibit Kidney Inflammation}

As expected, in model group, there were aberrant upregulated in the mRNA and protein expression levels of NLRP3, ASC, and caspase1 and the downstream cytokines 

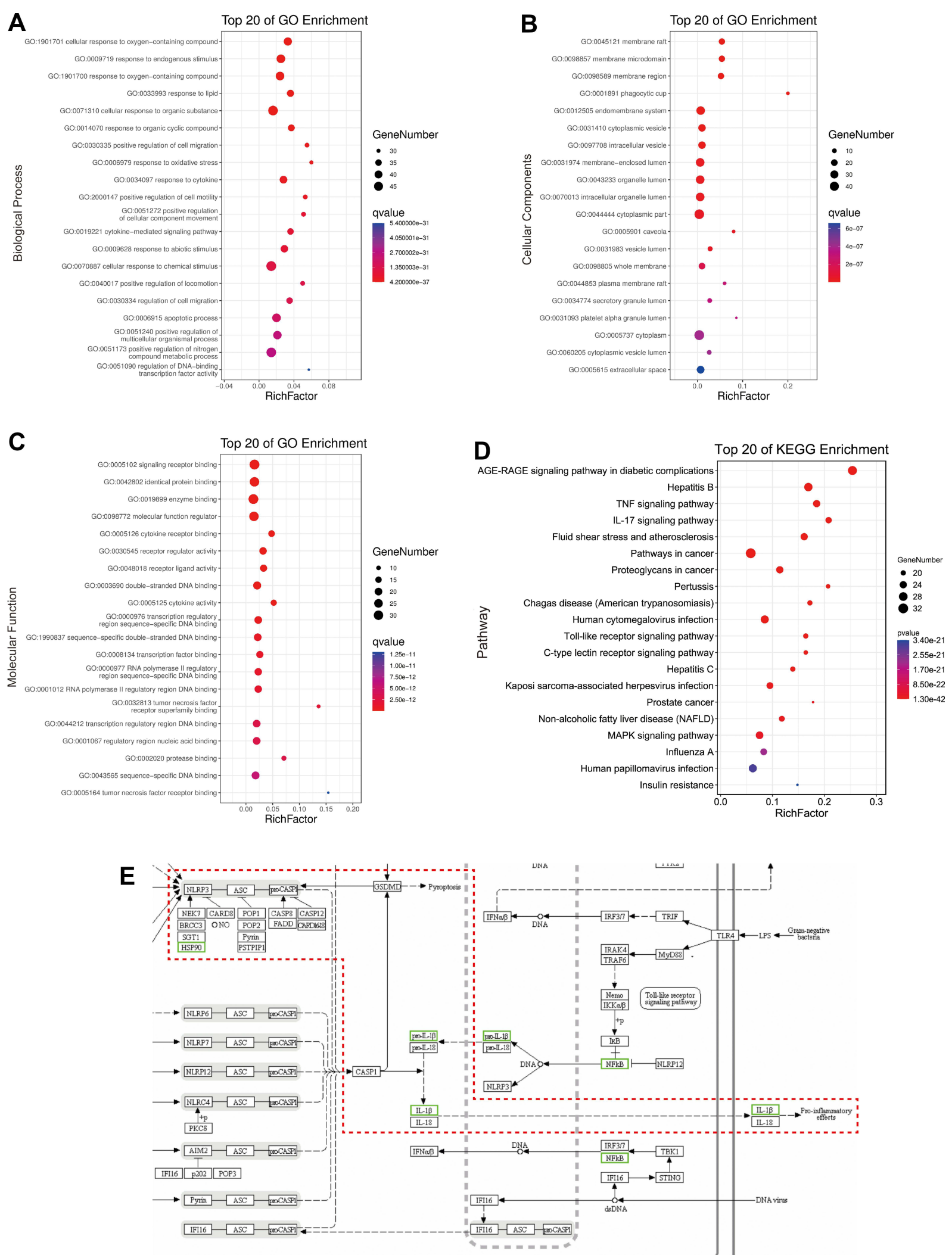

Figure 3 Go functional and KEGG pathway enrichment analysis. (A) Biological process enrichment. (B) Cellular components enrichment. (C) Molecular function enrichment. (D) KEGG pathway analysis. (E) NOD-like receptor signaling pathway (Red dotted rectangle: the key targets verified in this study). 

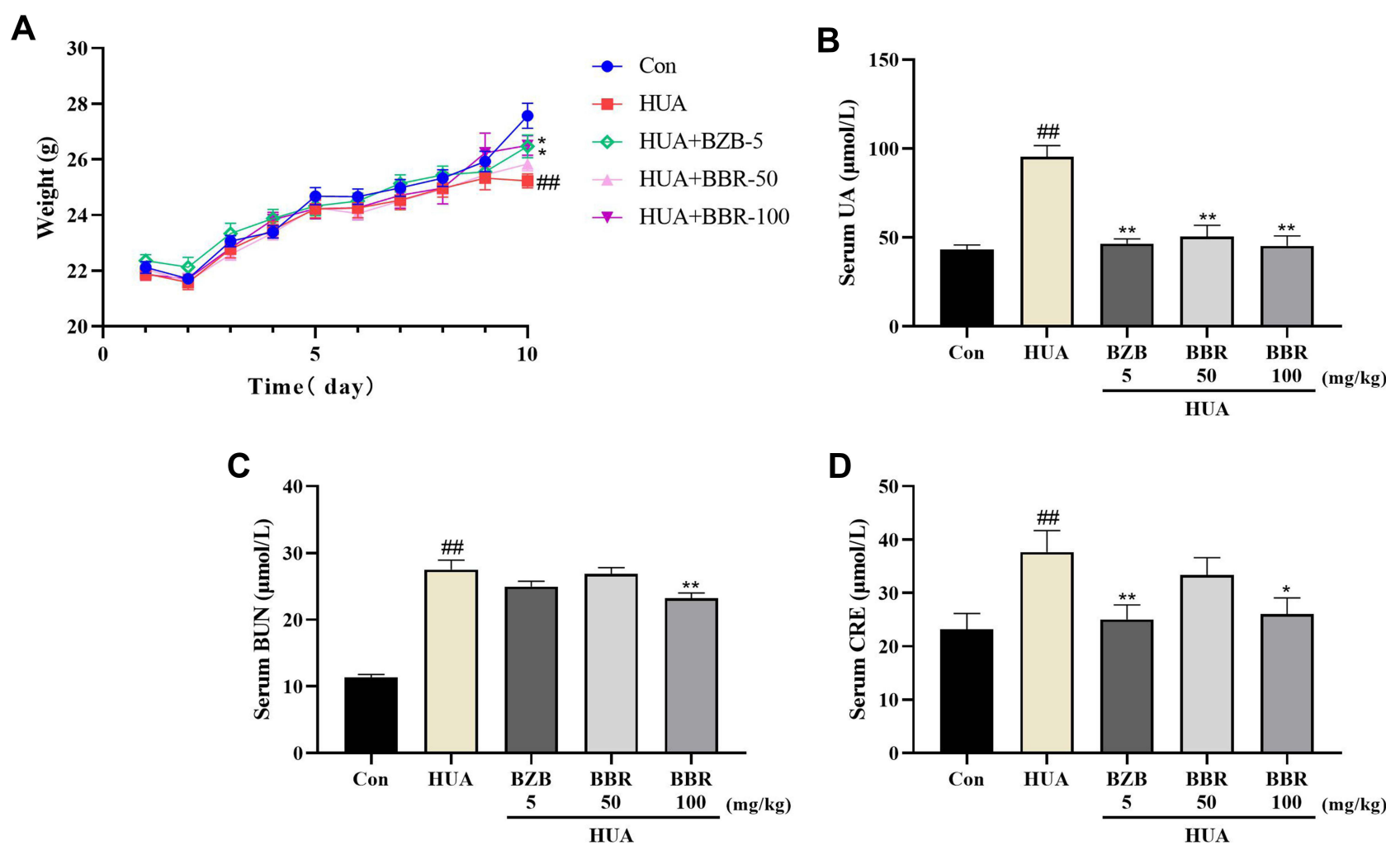

Figure 4 Anti-hyperuricemia and nephroprotective effects of BBR. (A) Body weight and serum levels of (B) UA, (C) BUN, and (D) CRE. All the results are shown as mean \pm SEM $(n=10) .{ }^{\#} p<0.01$ vs Con group. ${ }^{*} p<0.05$ and $* * p<0.01$ vs HUA group.

(IL-1 $\beta$ and IL-18). BZB and high-dose BBR significantly decreased the serum and kidney IL- $1 \beta$ and IL-18 levels compared to those in the model group (Figure 6A-D). Additionally, in the exploratory experiments, BZB and high-dose BBR significantly decreased the mRNA and protein expression levels of NLRP3, ASC, caspase1, and IL-1 $\beta$ (Figure 6E-M and the original images for all relevant Western blots were shown in Figures S1-S5). However, low-dose BBR had little impact regarding inhibiting NLRP3, as it only significantly changed caspase1 mRNA expression and ASC and IL-1 $\beta$ protein expression. In contrast, high-dose BBR exhibited obvious therapeutic effects regarding the suppression of the key molecules in the NLRP3 pathway, with no significant difference from the effects in the BZB group (the positive control group).

\section{BBR Corrected Renal Reabsorption Transporters Expression}

To further explore the effects of BBR on UA excretion in renal, we detected the mRNA and protein expression levels of URAT1 in vivo preliminarily and simulated the binding process of BBR and URAT1 protein in silico. The model mice had significantly increased mRNA and protein expression levels of
URAT1 (Figure 7B, D and E, the original images for all relevant Western blots were shown in Figures S6 and S7) compared to the Con group. BZB and high-dose BBR significantly reversed these trends. High-dose BBR had similar effects to BZB on URAT1 expression. The exploratory immunohistochemical analysis of URAT1 led to similar results (Figure 7A and C). URAT1 expression was up-regulated significantly in the proximal convoluted tubules of the model mice compared to the Con mice, but the expression was significantly inhibited by BZB and high-dose BBR.

The binding energy value for the BBR-URAT1 complex was $-8.10 \mathrm{kcal} / \mathrm{mol}$. Figure $7 \mathrm{~F}$ and $\mathrm{G}$ shows that $\mathrm{BBR}$ became inserted into the tunnel-shaped pocket, with various probable interactions. C- 8 on the iso-quinoline moiety donated a hydrogen atom to the ALA359 residue, forming a carbon H-bond. Additionally, another H-bond involving an oxygen atom of the dimethoxy and ALA359 was formed. A conventional H-bond was also observed between an oxygen atom in the methylenedioxy group and GLN245. Interestingly, there were three alkyl interactions, involving the pyridine ring plus LEU445 and PHE449 and the benzene ring plus ALA359. There were also three kinds of Pi-effect, involving a Pi-anion bond 


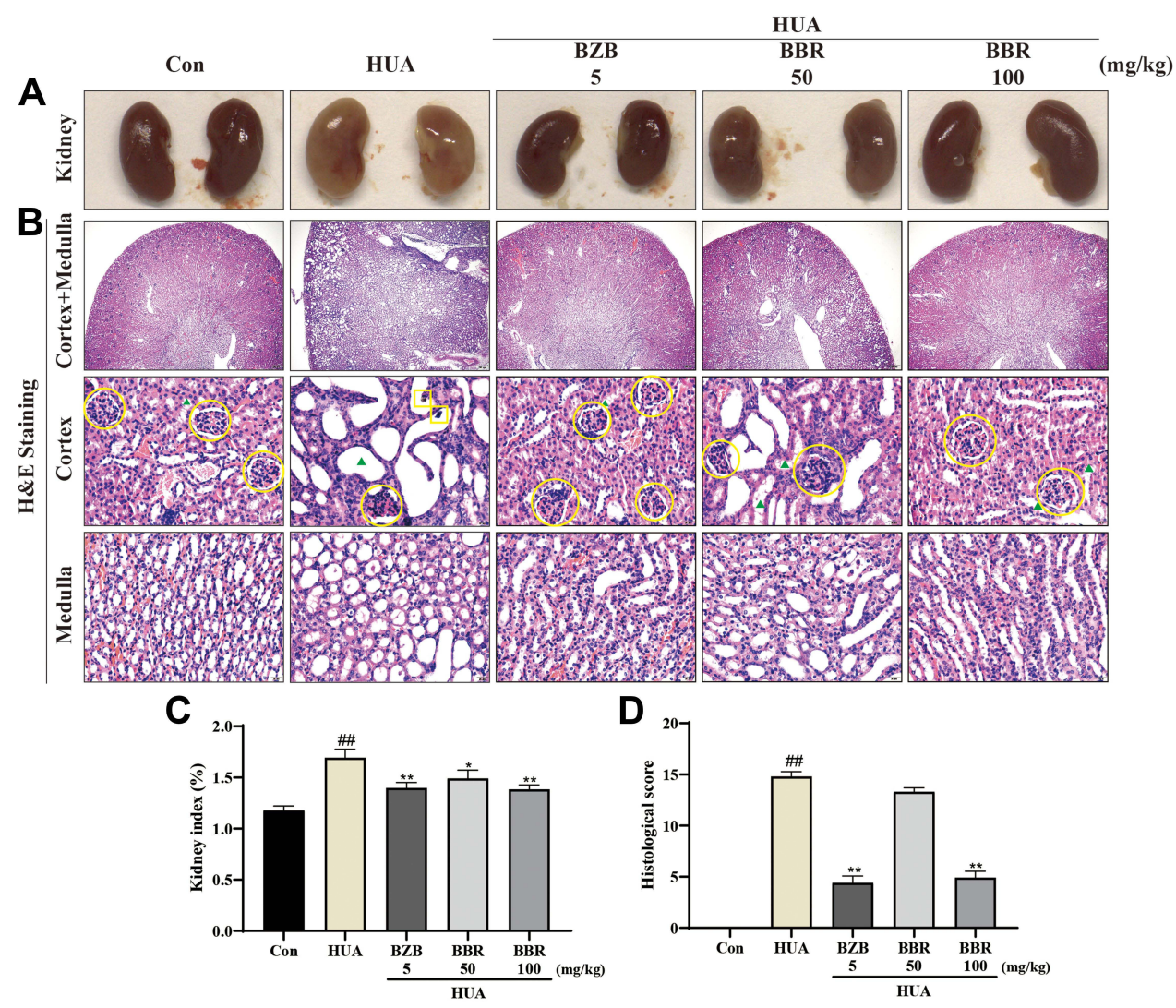

Figure 5 BBR improved kidney histology in model mice with PO- and HX-induced hyperuricemia. (A) Kidney surfaces. (B) H\&E-stained kidney sections: 40X panoramic images of cortex and medulla (scale bar, $200 \mu \mathrm{m}$ ); 400x images of cortex and medulla, respectively (scale bar, $20 \mu \mathrm{m}$ ). Green triangle, proximal convoluted tubule; yellow circle, glomeruli; yellow square, crystal kidney stones. (C) Relative kidney weight. (D) Histopathological scores of kidneys. All the results are shown as mean \pm SEM ( $n=10$ ). $\#^{2}<0.01$ vs Con group. ${ }^{*} p<0.05$ and $* * p<0.01$ vs HUA group.

with ASP389, a Pi-sigma bond with LEU445, and a Pi-Pi stacking interaction with PHE449. These chemical interactions demonstrate the promising inhibition of URAT1 by BBR.

\section{Discussion}

PC has been used to alleviate gout due to its functions of clearing heat and drying dampness in the theory of traditional Chinese medicine. ${ }^{16}$ From a pharmacological standpoint, these functions might be explained by its remarkable anti-inflammatory and anti-HUA effects, which are partly mediated by the alkaloids in $\mathrm{PC}^{42}$ BBR, a major alkaloid in PC, has significant antiinflammatory and nephroprotective effects against various injurious factors. Studies have reported the effects of BBR on certain transporters, indicating its potent anti-HUA effect. $^{25}$ Our bioinformatic analyses also showed that BBR could regulate at least 82 genes related to HUA and most of these genes enriched in the inflammatory signaling. In the present study, we aimed to verify the anti-
HUA effects of BBR and explore its potential mechanism in HUA mice.

Serum UA level is the hallmark of HUA. $^{43}$ Epidemiologic studies demonstrated that elevated UA increases the risk of renal disease, revealing that UA is not only a marker of kidney dysfunction but also a cause of kidney damage. ${ }^{3,4,44}$ BUN and CRE are major indicators of renal damage. ${ }^{45}$ The levels of BUN and CRE were significantly increased and there were severe histopathological changes (based on H\&E staining) in the model group. Based on these results and a previous report, ${ }^{43}$ a mouse model of HUA was successfully established in this study.

There was abundant clinical trials and laboratory studies demonstrating the ability of BBR to reduce UA and ameliorate kidney injury. Oral treatment with BBR $(300 \mathrm{mg} / \mathrm{d})$ for 60 days elicited a notable decrease in UA from 520.8 to 451.0 $\mu \mathrm{mol} / \mathrm{L} .{ }^{19}$ Another treatment combining silymarin to improve BBR's bioavailability was effective in lowing UA in obese patients with diabetes mellitus ${ }^{23}$ Compared with BZB alone, the association BBR + BZB could achieve superior hypouricemic effect. ${ }^{20} \mathrm{~A}$ recent research that HUA rates 

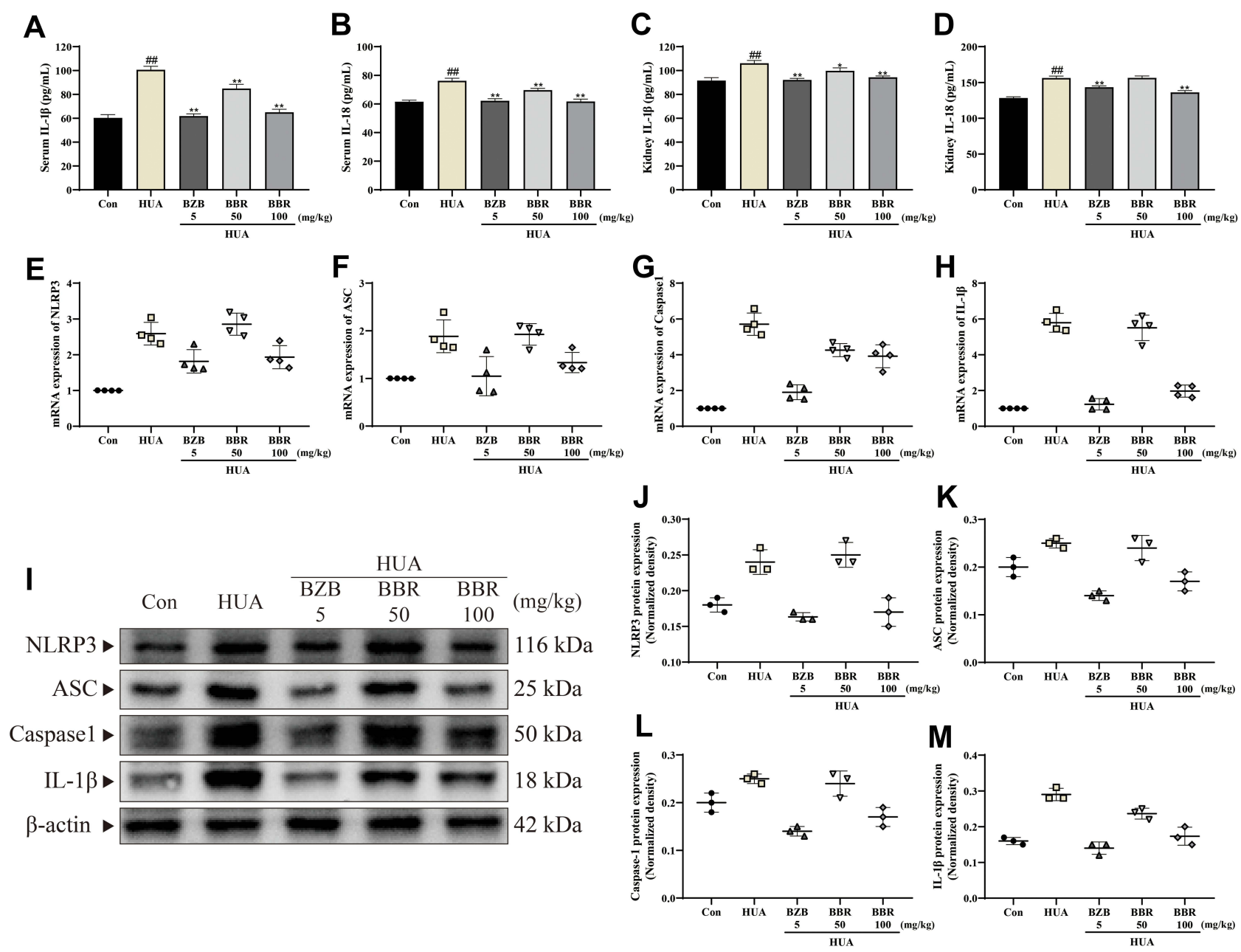

Figure 6 BBR suppressed NLRP3 inflammasome activation. Levels of (A) IL-I $\beta$ and (B) IL-I8 in serum. Levels of (C) IL-I $\beta$ and (D) IL-I8 in kidneys ( $\mathrm{n}=10$ ). mRNA expression levels of (E) NLRP3, (F) ASC, (G) caspasel, and (H) IL-I $\beta(n=4)$. (I) Representative Western blots of NLRP3, ASC, caspasel, and IL-I $\beta$ protein expression. Quantitative analysis of $(\mathbf{J}) \mathrm{NLRP3},(\mathbf{K})$ ASC, $(\mathbf{L})$ caspasel, and $(\mathbf{M})$ IL-I $\beta$ protein expression $(n=3)$. All the results are shown as mean \pm SEM. ${ }^{\#} \mathbf{P}<0.0 \mathrm{I}$ vs Con group. ${ }^{*} \mathrm{p}<0.05$ and $* * p<0.01$ vs HUA group.

were given BBR intraperitoneally also proved its remarkable anti-hyperuricemia action. ${ }^{23,24}$ In addition, BBR exhibited pronounced capability of improving kidney function in diabetes and atherosclerosis setting, ${ }^{29,30}$ which might be attributed to its anti-oxidant, anti-apoptotic, anti-inflammatory, anti-endoplasmic reticulum stress, and autophagy promotion effects. $^{28,29}$ In agreement with these studies, BBR significantly reversed the elevated UA and ameliorated the histopathological kidney injuries in our work. The nephroprotective property was further validated by decreased BUN and CRE after BBR treatment. These findings indicate the anti-HUA and renoprotective activity of BBR.

The immune response has a significant effect on the nephrotoxicity of $\mathrm{UA}^{46}$ In vivo and in vitro studies showed that UA activated the NLRP3 inflammasome and altered its downstream effectors. ${ }^{14}$ NLRP3 has been shown to be an essential regulator of renal inflammation and fibrosis in a mouse study. ${ }^{47}$ In the canonical pathway underlying the effects of NLRP3 activation, caspase 1 is activated, mature cytokines are secreted, and pyroptosis and renal necroinflammation are triggered. ${ }^{48}$ Research has linked IL-1 $\beta$ to acute and chronic kidney diseases ${ }^{49,50}$ and there is much evidence showing that it contributes to kidney damage. ${ }^{49}$ Inhibitors of IL-1 $\beta$ and IL-18 have become increasingly important nephroprotective therapeutic agents in various diseases. ${ }^{48,51}$

Our network pharmacology results indicated that the anti-HUA effect of BBR might be linked to its antiinflammatory activity. Many studies have shown that BBR suppresses NLRP3 activation and cytokine expression, including in the animal model involving monosodium UA crystal-induced inflammation. ${ }^{26,27}$ Additionally, 

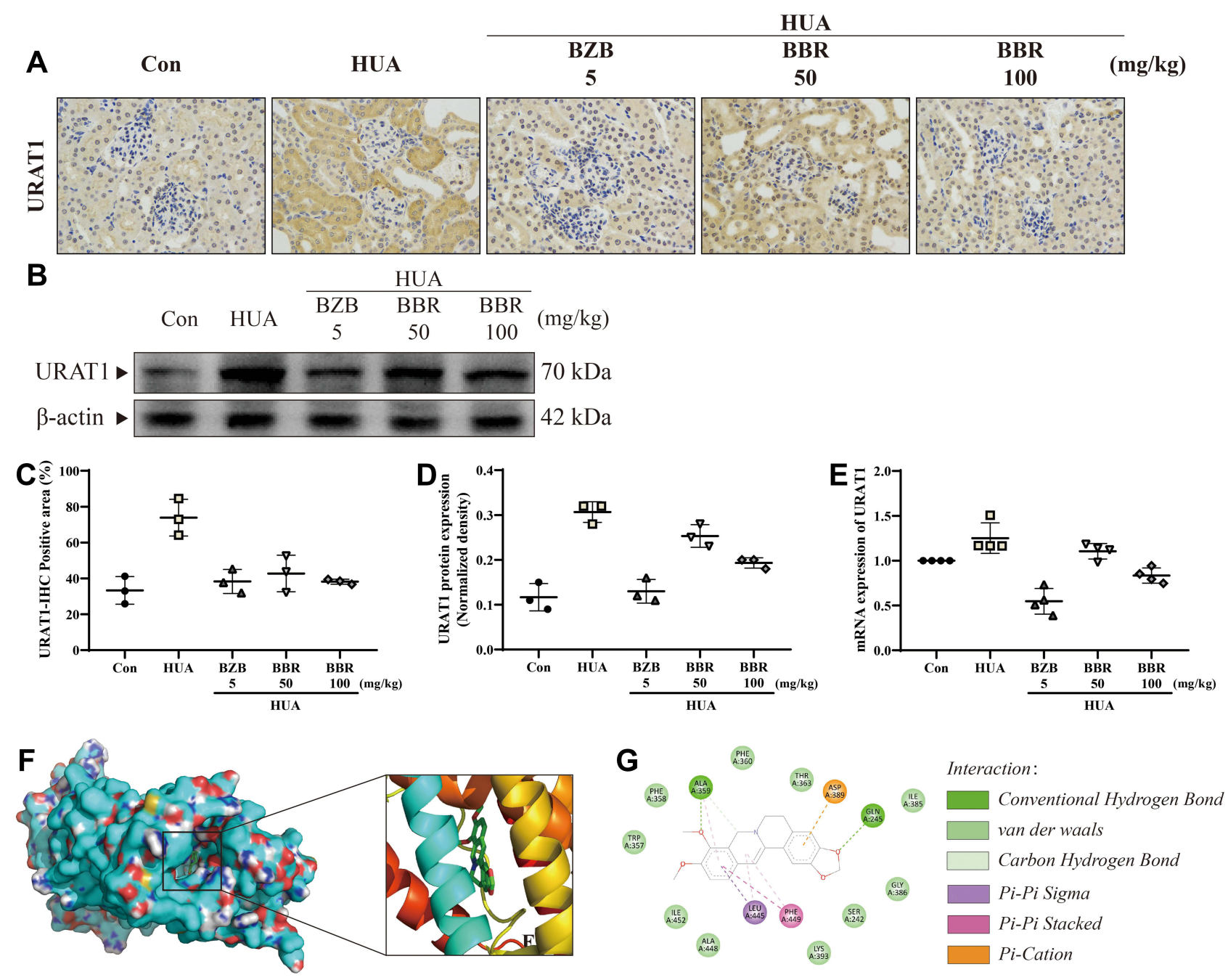

Figure 7 BBR improved UA excretion by regulating the mRNA and protein expression of URATI. (A) Kidney immunohistochemical staining (400x) and (B) representative Western blots of URATI. Quantitative analysis of $(\mathbf{C})$ immunohistochemical staining and (D) Western blots results $(n=3)$. $(\mathbf{E}) \mathrm{mRNA}$ expression level of URATI ( $\mathrm{n}=4)$. $(\mathbf{F})$ Initial orientation of the BBR in the URATI active pocket. (G) Chemical interactions between BBR and URATI. All the results are shown as mean \pm SEM.

in other inflammation-related models, such as diabetes mellitus, cancer, and gastroenteritis models, BBR exhibits significant anti-inflammatory activity by decreasing cytokine levels (such as IL-1 $\beta$ and IL-18), which might involve its inhibition of NLRP3. ${ }^{52}$ In our study, we found that BBR significantly reduced serum and kidney IL-1 $\beta$ and IL-18 levels, which concurs with previous reports. Thus, the effects of BBR on the NLRP3 inflammasome signaling pathway was further investigated. In line with the changes in cytokine secretion, BBR inhibited the expression of the downstream effectors of the NLRP3 inflammasome (ASC, caspase1, and IL-1 $\beta$ ), suggesting that the nephroprotective effects of BBR is partly attributed to the inactivation of NLRP3 inflammasome.

UA homoeostasis is largely determined by UA production, excretion, and reabsorption. XOD is a key enzyme for regulating the rate of UA synthesis. Hence, it has become a classical target for reducing UA overproduction. However, in vitro studies have shown that BBR only slightly inhibits XOD activity (inhibition rate $<50 \%$ ) and the half maximal inhibitory concentration $\left(\mathrm{IC}_{50}\right)$ is relatively high $(3.82 \mathrm{mg} / \mathrm{mL}){ }^{53,54}$ The results of our pilot study also agreed with these findings (data not shown), suggesting that the mechanism underlying the anti-HUA effect of BBR might not involve XOD.

URAT1, an organic anion transporter, is a major UA reabsorption transporter that is highly expressed in the proximal tubular epithelial cell membrane. ${ }^{55}$ It is an attractive target for new drug development for treating HUA. ${ }^{56}$ Although network pharmacology did not show the relationship between BBR and organic ion transporter, $\mathrm{BBR}$ shows promise as a URAT1 inhibitor. The $\mathrm{IC}_{50}$ 


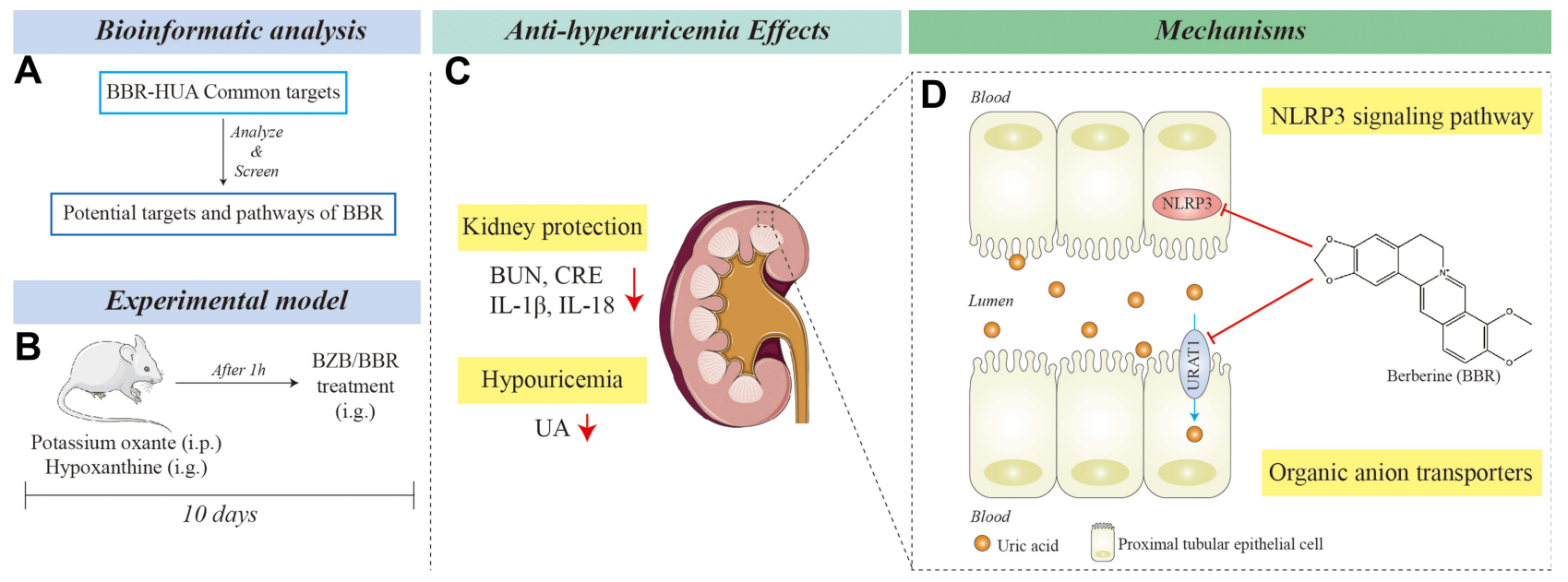

Figure 8 Schematic diagram showing the experiment design and results. (A) Network pharmacology analysis. (B) Experimental validation in a mouse model. (C) The antihyperuricemia and nephroprotective effects of berberine and (D) its potential mechanisms.

of BBR regarding inhibiting URAT1 is $13.6 \mu \mathrm{mol} / \mathrm{L}$, indicating the potential efficacy of BBR against URAT1. $^{25}$ In our study, BBR inhibited URAT1 mRNA and protein expression levels, which indicates that the inhibitory effect of $\mathrm{BBR}$ on reabsorption transporters may underlie its anti-HUA effect. We also performed a virtual docking analysis to predict the affinity and interactions between the candidate compound (BBR) and the target protein (URAT1). The in silico observation regarding the strong affinity between BBR and URAT1 was attributed to multiple chemical bonds.

\section{Conclusion}

In summary, this pioneering study (Figure 8A, B) on the anti-HUA and nephroprotective effects of BBR in a mouse model of PO- and HX-induced HUA showed that BBR effectively reduces the UA level and ameliorates the kidney damage (Figure 8C). These effects are associated with the regulation of the NLRP3 inflammasome and UA transporters (Figure 8D). Our findings provide evidence that BBR might be useful as an alternative uricosuric drug for treating HUA. It is worthwhile to further investigate the underlying mechanism in more detail and the efficacy of BBR in alternative models of HUA.

\section{Acknowledgments}

This research was supported by the National Science Foundation of China (No. 82074082), National Natural Science Foundation of Guangdong (Nos. 2021A1515011490 \& 2019A1515010819), Key Program for Subject Research of Guangzhou University of Chinese
Medicine (No. XK2019002) and Characteristic Cultivation Program for Subject Research of Guangzhou University of Chinese Medicine (No. XKP2019007).

\section{Disclosure}

The authors report no conflicts of interest in this work.

\section{References}

1. Terkeltaub R. Update on gout: new therapeutic strategies and options. Nat Rev Rheumatol. 2010;6(1):30-38. doi:10.1038/nrrheum.2009.236

2. So A, Thorens B. Uric acid transport and disease. J Clin Invest. 2010;120(6):1791-1799. doi:10.1172/JCI42344

3. Russo E, Viazzi F, Pontremoli R, et al. Association of uric acid with kidney function and albuminuria: the Uric Acid Right for heArt Health (URRAH) project. J Nephrol. 2021. doi:10.1007/s40620-021-00985-4

4. Hahn K, Kanbay M, Lanaspa MA, Johnson RJ, Ejaz AA. Serum uric acid and acute kidney injury: a mini review. $J$ Adv Res. 2017;8 (5):529-536. doi:10.1016/j.jare.2016.09.006

5. Maloberti A, Giannattasio C, Bombelli M, et al. Hyperuricemia and risk of cardiovascular outcomes: the experience of the URRAH (Uric Acid Right for Heart Health) project. High Blood Press Cardiovasc Prev. 2020;27(2):121-128. doi:10.1007/s40292-020-00368-z

6. Rock KL, Kataoka H, Lai JJ. Uric acid as a danger signal in gout and its comorbidities. Nat Rev Rheumatol. 2013;9(1):13-23. doi:10.1038/ nrrheum.2012.143

7. $\mathrm{Lu} \mathrm{W}, \mathrm{Xu} \mathrm{Y}$, Shao $\mathrm{X}$, et al. Uric acid produces an inflammatory response through activation of NF-kappaB in the hypothalamus: implications for the pathogenesis of metabolic disorders. Sci Rep. 2015;5:12144. doi:10.1038/srep12144

8. Desai J, Steiger S, Anders HJ. Molecular pathophysiology of gout. Trends Mol Med. 2017;23(8):756-768. doi:10.1016/j.molmed.20 17.06.005

9. Maiuolo J, Oppedisano F, Gratteri S, Muscoli C, Mollace V. Regulation of uric acid metabolism and excretion. Int $J$ Cardiol. 2016;213:8-14. doi:10.1016/j.ijcard.2015.08.109

10. Wang CW, Dao RL, Chung WH. Immunopathogenesis and risk factors for allopurinol severe cutaneous adverse reactions. Curr Opin Allergy Clin Immunol. 2016;16(4):339-345. doi:10.1097/ ACI.0000000000000286 
11. Harrold L. New developments in gout. Curr Opin Rheumatol. 2013;25(3):304-309. doi:10.1097/BOR.0b013e32835fd5e5

12. Becker MA, Schumacher HR, Espinoza LR, et al. The urate-lowering efficacy and safety of febuxostat in the treatment of the hyperuricemia of gout: the CONFIRMS trial. Arthritis Res Ther. 2010;12(2): R63. doi: $10.1186 /$ ar2978

13. Mackenzie IS, Ford I, Nuki G, et al. Long-term cardiovascular safety of febuxostat compared with allopurinol in patients with gout (FAST): a multicentre, prospective, randomised, open-label, non-inferiority trial. Lancet (London, England). 2020;396 (10264):1745-1757. doi:10.1016/S0140-6736(20)32234-0

14. Bardin T, Richette P. FAST: new look at the febuxostat safety profile. Lancet (London, England). 2020;396(10264):1704-1705. doi:10.1016/S0140-6736(20)32343-6

15. Wang X, Wang Y-G. Progress in treatment of gout using chinese and western medicine. Chin J Integr Med. 2020;26(1):8-13. doi:10.1007/ s11655-019-3058-y

16. Chi X, Zhang H, Zhang S, Ma K. Chinese herbal medicine for gout: a review of the clinical evidence and pharmacological mechanisms. Chin Med. 2020;15:17. doi:10.1186/s13020-020-0297-y

17. Zhao F, Guochun L, Yang Y, Shi L, Xu L, Yin L. A network pharmacology approach to determine active ingredients and rationality of herb combinations of Modified-Simiaowan for treatment of gout. $J$ Ethnopharmacol. 2015;168:1-16. doi:10.1016/j. jep.2015.03.035

18. Zhang H, Wei J, Xue R, et al. Berberine lowers blood glucose in type 2 diabetes mellitus patients through increasing insulin receptor expression. Metabolism. 2010;59(2):285-292. doi:10.1016/j. metabol.2009.07.029

19. Wu LG, Chen M, Wu Y. Effect of berberine on hyperuricemia. Chin $J$ Integr Tradit West Med. 2010;30(6):659-662.

20. Wang Y, Yang R, Shan L, Yuan J. Efficacy and safety of berberine combined with benzbromarone in the treatment of hyperuricemia with gout. Chin J Mod Drug Appl. 2018;12(7):3-6.

21. Cai SW. Clinical effect and safety of combination medicine for elderly patients with hypertension and gout. China Reflexolocy. 2017;26(11):84-89.

22. Huang GL. Effect of amlodipine and berberine in mild and midrange hypertension patients complicated with hyperuricemia arthrolithiasis. China Pharm. 2013;22(5):32-33.

23. Guarino G, Strollo F, Carbone L, et al. Bioimpedance analysis, metabolic effects and safety of the association Berberis aristata/ Bilybum marianum: a 52-week double-blind, placebo-controlled study in obese patients with type 2 diabetes. J Biol Regul Homeost Agents. 2017;31(2):495-502.

24. Naz H, Naz S, Miraj R, et al. The effect of berberine, a drug from chinese folk medicine, on serum and urinary uric acid levels in rats with hyperuricemia. Cureus. 2021;13(2):e13186.

25. Weidang W, Xingyan Z, Zihong W, et al. Inhibition of berberine on organ anion transporters and its bidirectional trans-membrane transport. Drug Eval Res. 2017;40(6):778-782.

26. Liu YF, Wen CY, Chen Z, Wang Y, Huang Y, Tu SH. Effects of berberine on NLRP3 and IL-1beta expressions in monocytic THP-1 cells with monosodium urate crystals-induced inflammation. Biomed Res Int. 2016;2016:2503703.

27. Dinesh P, Rasool M. Berberine, an isoquinoline alkaloid suppresses TXNIP mediated NLRP3 inflammasome activation in MSU crystal stimulated RAW 264.7 macrophages through the upregulation of $\mathrm{Nrf} 2$ transcription factor and alleviates MSU crystal induced inflammation in rats. Int Immunopharmacol. 2017;44:26-37. doi:10.1016/j. intimp.2016.12.031

28. Ni WJ, Ding HH, Tang LQ. Berberine as a promising anti-diabetic nephropathy drug: an analysis of its effects and mechanisms. Eur J Pharmacol. 2015;760:103-112. doi:10.1016/ j.ejphar.2015.04.017
29. Wan X, Chen X, Liu L, et al. Berberine ameliorates chronic kidney injury caused by atherosclerotic renovascular disease through the suppression of nfikb signaling pathway in rats. PLoS One. 2013;8 (3):e59794. doi:10.1371/journal.pone.0059794

30. Li Z, Zhang W. Protective effect of berberine on renal fibrosis caused by diabetic nephropathy. Mol Med Rep. 2017;16(2):1055-1062. doi: $10.3892 / \mathrm{mmr} .2017 .6707$

31. Yong T, Li D, Li M, et al. Anti-hyperuricemic effect of 2-Hydroxy4-methoxy-benzophenone-5-sulfonic acid in hyperuricemic mice through XOD. Molecules. 2018;23:10. doi:10.3390/molecules2 3102671

32. Animal Care Committee. Rodent Anesthesia and Analgesia Formulary and General Drug Information; 2016. Available from: https://animalcare.ubc.ca/sites/default/files/documents/Guideline $\%$ 20-\%20Rodent $\% 20$ Anesthesia\%20Analgesia\%20Formulary\%20\% 282016\%29.pdf. Accessed May 30, 2021.

33. Russ M, Ott S, Bedarf JR, Kirschfink M, Hiebl B, Unger JK. Increased compensatory kidney workload results in cellular damage in a short time porcine model of mixed acidemia - Is acidemia a 'first hit' in acute kidney injury? PLoS One. 2019;14(6):e0218308. doi:10.1016/j.semarthrit.2020.04.007

34. Bustin SA, Benes V, Garson JA, et al. The MIQE guidelines: minimum information for publication of quantitative real-time PCR experiments. Clin Chem. 2009;55(4):611-622. doi:10.1373/ clinchem.2008.112797

35. Mitchell J. RT-PCR Protocols. Methods in Molecular Biology, Vol. 193: O’Connell J, ed. Humana Press, 2002. ISBN 089603875 0. J Clin Pathol. 2003;56(5):400. doi:10.1136/jcp.56.5.400-a

36. Mahmood T, Yang P-C. Western blot: technique, theory, and trouble shooting. N Am J Med Sci. 2012;4(9):429-434. doi:10.4103/19472714.100998

37. Dou Y, Huang R, Li Q, et al. Oxyberberine, an absorbed metabolite of berberine, possess superior hypoglycemic effect via regulating the PI3K/Akt and Nrf2 signaling pathways. Biomed Pharmacother. 2021;137:111312. doi:10.1016/j.biopha.2021.111312

38. Syrbu SI, Cohen MB. An enhanced antigen-retrieval protocol for immunohistochemical staining of formalin-fixed, paraffin-embedded tissues. Methods Mol Biol. 2011;717:101-110.

39. Zhou J, Wang T, Dou Y, et al. Brusatol ameliorates 2, 4, 6-trinitrobenzenesulfonic acid-induced experimental colitis in rats: involvement of NF- $\mathrm{KB}$ pathway and NLRP3 inflammasome. Int Immunopharmacol. 2018;64:264-274. doi:10.1016/j. intimp.2018.09.008

40. Forli S, Huey R, Pique ME, Sanner MF, Goodsell DS, Olson AJ. Computational protein-ligand docking and virtual drug screening with the AutoDock suite. Nat Protoc. 2016;11(5):905-919. doi:10.1038/nprot.2016.051

41. Curtis MJ, Alexander S, Cirino G, et al. Experimental design and analysis and their reporting II: updated and simplified guidance for authors and peer reviewers. Br J Pharmacol. 2018;175(7):987-993. doi:10.1111/bph.14153

42. Sun Y, Lenon GB, Yang AWH. Phellodendri cortex: a phytochemical, pharmacological, and pharmacokinetic review. Evid Based Complement Alternat Med. 2019;2019:7621929. doi:10.1155/2019/7621929

43. Lu J, Dalbeth N, Yin H, Li C, Merriman TR, Wei WH. Mouse models for human hyperuricaemia: a critical review. Nat Rev Rheumatol. 2019;15(7):413-426. doi:10.1038/s41584-019-0222-x

44. Obermayr RP, Temml C, Gutjahr G, Knechtelsdorfer M, Oberbauer R, Klauser-Braun R. Elevated uric acid increases the risk for kidney disease. J Am Soc Nephrol. 2008;19(12):2407-2413. doi:10.1681/ASN.2008010080

45. Kirtane AJ, Leder DM, Waikar SS, et al. Serum blood urea nitrogen as an independent marker of subsequent mortality among patients with acute coronary syndromes and normal to mildly reduced glomerular filtration rates. $J$ Am Coll Cardiol. 2005;45(11):1781-1786. doi:10.1016/j.jacc.2005.02.068 
46. Jung SW, Kim SM, Kim YG, Lee SH, Moon JY. Uric acid and inflammation in kidney disease. Am J Physiol Renal Physiol. 2020;318(6):F1327-f1340. doi:10.1152/ajprenal.00272.2019

47. Bakker PJ, Butter LM, Kors L, et al. Nlrp3 is a key modulator of diet-induced nephropathy and renal cholesterol accumulation. Kidney Int. 2014;85(5):1112-1122. doi:10.1038/ki.2013.503

48. Komada T, Muruve DA. The role of inflammasomes in kidney disease. Nat Rev Nephrol. 2019;15(8):501-520.

49. Nowak KL, Chonchol M, Ikizler TA, et al. IL-1 inhibition and vascular function in CKD. J Am Soc Nephrol. 2017;28(3):971-980. doi:10.1681/ASN.2016040453

50. Wu H, Craft ML, Wang P, et al. IL-18 contributes to renal damage after ischemia-reperfusion. $J$ Am Soc Nephrol. 2008;19 (12):2331-2341. doi:10.1681/ASN.2008020170

51. Afsar B, Covic A, Ortiz A, Afsar RE, Kanbay M. The future of IL-1 targeting in kidney disease. Drugs. 2018;78(11):1073-1083. doi:10.1007/s40265-018-0942-2

52. Li Z, Geng Y-N, Jiang J-D, Kong W-J. Antioxidant and anti-inflammatory activities of berberine in the treatment of diabetes mellitus. Evid Based Compl Altern Med. 2014;2014:289264.
53. Li S, Liu C, Guo L, et al. Ultrafiltration liquid chromatography combined with high-speed countercurrent chromatography for screening and isolating potential alpha-glucosidase and xanthine oxidase inhibitors from Cortex Phellodendri. J Sep Sci. 2014;37 (18):2504-2512. doi:10.1002/jssc.201400475

54. Kong LD, Cai Y, Huang WW, Cheng CH, Tan RX. Inhibition of xanthine oxidase by some Chinese medicinal plants used to treat gout. J Ethnopharmacol. 2000;73(1-2):199-207. doi:10.1016/ S0378-8741(00)00305-6

55. Keenan RT. The biology of urate. Semin Arthritis Rheum. 2020;50 (3s):S2-s10

56. Dong Y, Zhao T, Ai W, et al. Novel urate transporter 1 (URAT1) inhibitors: a review of recent patent literature (2016-2019). Expert Opin Ther Pat. 2019;29(11):871-879. doi:10.1080/13543776.20 19.1676727

\section{Publish your work in this journal}

Drug Design, Development and Therapy is an international, peerreviewed open-access journal that spans the spectrum of drug design and development through to clinical applications. Clinical outcomes, patient safety, and programs for the development and effective, safe, and sustained use of medicines are a feature of the journal, which has also been accepted for indexing on PubMed Central. The manuscript management system is completely online and includes a very quick and fair peer-review system, which is all easy to use. Visit http://www. dovepress.com/testimonials.php to read real quotes from published authors. 